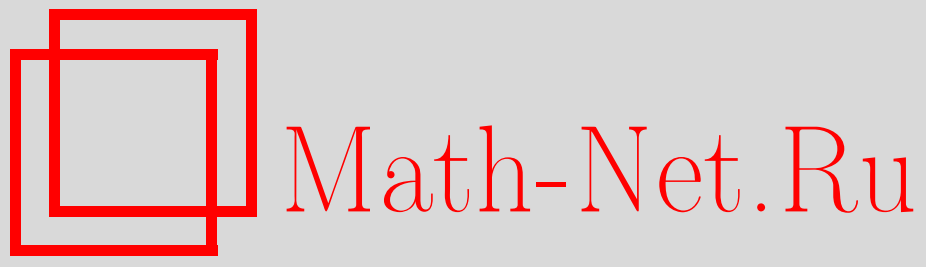

В. Д. Седых, Полная система линейных соотношений между эйлеровыми характеристиками многообразий особенностей коранга 1 фронта общего положения, Функи. анализ и его прил., 2004, том 38, выпуск $4,73-78$

DOI: https://doi.org/10.4213/faa127

Использование Общероссийского математического портала MathNet.Ru подразумевает, что вы прочитали и согласны с пользовательским соглашением

http://www.mathnet.ru/rus/agreement

Параметры загрузки:

IP : 54.157 .27 .8

26 апреля 2023 г., 16:11:48

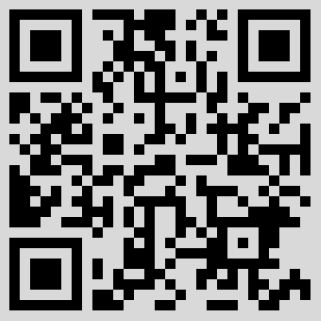


Функииональньй анализ и его приложения

2004, т. 38 , вып. 4, с. 73-78

УДК 515.16

\title{
Полная система линейных соотношений между эйлеровыми характеристиками многообразий особенностей коранга 1 фронта общего положения*
}

\author{
(C) 2004. В. Д. СЕдых
}

1. Введение. Рассмотрим фронт $\mathscr{F}$ общего положения в $C^{\infty}$-гладком $n$-мерном многообразии $V$. Это особая гиперповерхность, являющаяся образом (собственного) лежандрова отображения $f: L \rightarrow V$ гладкого $(n-1)$-мерного многообразия $L$. Образ каждой связной компоненты многообразия $L$ называется неприводимой компонентой фронта $\mathscr{F}$. Фронт $\mathscr{F}$ связного многообразия $L$ называется неприводимым.

Если лежандрово отображение $f$ устойчиво и размерность ядра его первого дифференциала ни в одной точке не превосходит 1 , то фронт $\mathscr{F}$ называется устойчивым фронтом коранга 1. Особенности (ростки) такого фронта классифицируются по элементам $A_{\mu_{1}}+\cdots+A_{\mu_{m}}$ свободной абелевой полугруппы по сложению с образующими $A_{1}, A_{2}, \ldots$ (см. [1]).

ОПРеДЕЛЕНИЕ. Фронт $\mathscr{F}$ имеет особенность типа $A_{\mu}$ в точке $x$, если его росток $(\mathscr{F}, x)$ в этой точке диффеоморфен ростку в нуле гиперповерхности в $\mathbb{R}^{n}=\left\{\left(\lambda_{0}, \ldots, \lambda_{n-1}\right)\right\}$, образованной точками, в которых многочлен

$$
t^{\mu+1}+\lambda_{\mu-1} t^{\mu-1}+\cdots+\lambda_{1} t+\lambda_{0}
$$

имеет кратный вещественный корень.

Множество особенностей типа $A_{\mu}$ фронта $\mathscr{F}$ (т.е. множество точек, в которых $\mathscr{F}$ имеет особенность этого типа) является гладким подмногообразием коразмерности $\mu$ в $V$.

ОПРЕДЕЛЕНИЕ. Фронт $\mathscr{F}$ имеет особенность типа $\mathscr{A}=A_{\mu_{1}}+\cdots+A_{\mu_{m}}$ в точке $x$, если росток $(\mathscr{F}, x)$ состоит из $m$ неприводимых компонент, которые имеют (как ростки фронтов) особенности типов $A_{\mu_{1}}, \ldots, A_{\mu_{m}}$ в точке $x$, причем ростки многообразий особенностей этих типов на соответствующих компонентах пересекаются в точке $x$ трансверсально.

Числа $\mu_{1}, \ldots, \mu_{m}$ называются кратностями особенности типа $\mathscr{A}$. Их сумма $\operatorname{codim} \mathscr{A}=\mu_{1}+\cdots+\mu_{m}$ называется коразмерностъю этой особенности. Коразмерность особенности не превышает размерности $n$ объемлющего многообразия $V$. Точки фронта $\mathscr{F}$, в которых он имеет особенность типа $\mathscr{A}$, образуют гладкое (вообще говоря, незамкнутое) подмногообразие $\mathscr{A}_{\mathscr{F}}$ коразмерности $\operatorname{codim} \mathscr{A}$ в $V$. Топологическую эйлерову характеристику этого многообразия мы будем обозначать через $\chi\left(\mathscr{A}_{\mathscr{F}}\right)$.

\footnotetext{
*Работа частично поддержана грантами РФФИ-02-01-00655 и НШ-1972.2003.1.
} 
Tеорема 1 [4]. Пусть $\mathscr{F}$ - компактный устойчивый фронт коранга 1 в гладком n-мерном многообразии $V$. Тогда для любого $\mathscr{A}=A_{\mu_{1}}+\cdots+A_{\mu_{m}}, m_{\text {- }}$ кого, что многообразие $\mathscr{A}_{\mathscr{F}}$ особенностей типа $\mathscr{A}$ фронта $\mathscr{F}$ имеет нечетную размерность, эйлерова характеристика $\chi\left(\mathscr{A}_{\mathscr{F}}\right)$ многообразия $\mathscr{A}_{\mathscr{F}}$ является линейной комбинацией,

$$
\chi\left(\mathscr{A}_{\mathscr{F}}\right)=\sum_{X} K_{\mathscr{A}}(X) \chi\left(X_{\mathscr{F}}\right)
$$

эйлеровых характеристик $\chi\left(X_{\mathscr{F}}\right)$ четномерных многообразий $X_{\mathscr{F}}$ особенностей типов $X$ фронта $\mathscr{F}$, где $\operatorname{codim} X>\operatorname{codim} \mathscr{A}$. Эта комбинация универсальна в том смысле, что каждый ее коэффициент $K_{\mathscr{A}}(X)$ зависит только oт $\mathscr{A}$ и $X$ и не зависит от $n, V$ и $\mathscr{F}$.

Например, для особенностей коразмерности 2 (их всего две) соответствующие формулы имеют вид

$$
\begin{aligned}
\chi\left(2 A_{1}\right)=3 \chi & \left(3 A_{1}\right)+\chi\left(A_{2}+A_{1}\right)+\frac{1}{2} \chi\left(A_{3}\right)-20 \chi\left(5 A_{1}\right) \\
& -9 \chi\left(A_{2}+3 A_{1}\right)-4 \chi\left(2 A_{2}+A_{1}\right)-\frac{11}{2} \chi\left(A_{3}+2 A_{1}\right) \\
& -\frac{5}{2} \chi\left(A_{3}+A_{2}\right)-\frac{7}{2} \chi\left(A_{4}+A_{1}\right)-2 \chi\left(A_{5}\right)+\ldots \\
\chi\left(A_{2}\right)=\chi & \left(A_{2}+A_{1}\right)+\chi\left(A_{3}\right)-2 \chi\left(A_{2}+3 A_{1}\right)-2 \chi\left(2 A_{2}+A_{1}\right) \\
& -2 \chi\left(A_{3}+2 A_{1}\right)-\frac{3}{2} \chi\left(A_{3}+A_{2}\right)-2 \chi\left(A_{4}+A_{1}\right)-2 \chi\left(A_{5}\right)+\ldots
\end{aligned}
$$

Здесь $n$ нечетное, индекс $\mathscr{F}$ в обозначениях эйлеровых характеристик соответствующих многообразий опущен (для простоты), а многоточие обозначает линейную комбинацию эйлеровых характеристик многообразий $X_{\mathscr{F}}$ особенностей типов $X$ нечетных коразмерностей, начиная с 7 -й.

Работа [4] доставляет простой комбинаторный алгоритм для вычисления всех соотношений вида $(*)$ в любой размерности $n$. Ниже доказано, что в условиях теоремы 1 не существует универсальных линейных соотношений (с вещественными коэффициентами) между эйлеровыми характеристиками многообразий особенностей фронта $\mathscr{F}$, которые не были бы линейными комбинациями соотношений $(*)$.

2. Формулировка результата. Составим список $\mathscr{A}^{1}, \ldots, \mathscr{A}^{N}$ классов особенностей типов $A_{\mu_{1}}+\cdots+A_{\mu_{m}}$, которые могут быть у фронтов в гладком $n$-мерном многообразии и коразмерность которых сравнима с $n$ по модулю 2 (многообразия таких особенностей имеют четную размерность). Каждому компактному устойчивому фронту $\mathscr{F}$ коранга 1 в $\mathbb{R}^{n}$ сопоставим вектор

$$
v(\mathscr{F})=\left(\chi\left(\mathscr{A}_{\mathscr{F}}^{1}\right), \ldots, \chi\left(\mathscr{A}_{\mathscr{F}}^{N}\right)\right) \in \mathbb{R}^{N}
$$

из эйлеровых характеристик $\chi\left(\mathscr{A}_{\mathscr{F}}^{i}\right)$ многообразий $\mathscr{A}_{\mathscr{F}}^{i}$ особенностей типов $\mathscr{A}^{i}$, $i=1, \ldots, N$.

Пусть $G$ - подмножество в $\mathbb{R}^{N}$, образованное векторами $v(\mathscr{F})$ для всех фронтов $\mathscr{F}$ в $\mathbb{R}^{n}$ указанного типа. Подмножество в $G$, образованное векторами $v(\mathscr{F})$ для неприводимых фронтов $\mathscr{F}$, обозначим через $G_{0}$.

Теорема 2. Множество $G$ содержит полугруппу с $N$ линейно независимыми образующими. Если $n>1$, то это верно и для множества $G_{0}$. 
Отсюда следует, что теорема 1 описывает полную систему универсальных линейных соотношений между эйлеровыми характеристиками многообразий особенностей компактных устойчивых фронтов коранга 1. Это означает следующее.

Предположим, что список всех классов особенностей типов $A_{\mu_{1}}+\cdots+A_{\mu_{m}}$, которые могут быть у $(n-1)$-мерных фронтов, состоит из $p$ элементов. Рассмотрим подмножество в $\mathbb{R}^{p}$, образованное (упорядоченными) наборами эйлеровых характеристик $\chi\left(\mathscr{A}_{\mathscr{F}}\right)$ многообразий $\mathscr{A}_{\mathscr{F}}$ особенностей типов $\mathscr{A}$ из этого списка у компактных устойчивых фронтов $\mathscr{F}$ коранга 1 в $n$-мерном пространстве. Тогда система соотношений вида (*) задает минимальное аффинное подпространство в $\mathbb{R}^{p}$, содержащее указанное подмножество.

ЗАмЕЧАнИЕ. Эйлеровы характеристики многообразий особенностей фронта ограничены также условиями типа неравенства, четности и т. п. (см. [3]). Однако мы не знаем всех этих условий.

3. Вспомогательные утверждения. Прежде чем доказывать теорему 2, мы построим некоторые специальные фронты в евклидовом пространстве $\mathbb{R}^{n}$.

ПРЕДЛОЖЕНИЕ 1. Пусть $\mathscr{F}$ - компактный устойчивый фронт коранга 1 в $\mathbb{R}^{n}$, где $n>1$. Предположим, что этот бронт имеет ровно $k$ неприводимых компонент. Тогда существует неприводимый компактный устойчивый фронт $\widetilde{\mathscr{F}}$ коранга 1 в $\mathbb{R}^{n}$, такой, что

(1) многообразие $\mathscr{A}_{\widetilde{F}}$ особенностей типа $\mathscr{A}$ фронта $\widetilde{\mathscr{F}}$ совпадает с многообразием $\mathscr{A}_{\mathscr{F}}$ особенностей типа $\mathscr{A}$ фронта $\mathscr{F}$ для любого $\mathscr{A} \neq A_{1}$;

(2) эйлерова характеристика многообразия $\left(A_{1}\right)_{\mathscr{F}}$ особенностей типа $A_{1}$ фронта $\widetilde{\mathscr{F}}$ меньше эйлеровой характеристики многообразия $\left(A_{1}\right) \mathscr{F}$ особенностей типа $A_{1}$ фронта $\mathscr{F}$ на $k-1$ эйлеровых характеристик $(n-1)$-мерной сферъь.

Доказательство. Рассмотрим $C^{\infty}$-вложенную кривую $\gamma:[a, b] \subset \mathbb{R} \rightarrow \mathbb{R}^{n}$, которая пересекает все неприводимые компоненты фронта $\mathscr{F}$, причем только в его гладких точках и только трансверсально $(\gamma(a), \gamma(b) \notin \mathscr{F})$. Нумерация моментов $t \in(a, b)$ первого пересечения кривой $\gamma$ с неприводимыми компонентами фронта $\mathscr{F}$ в порядке их возрастания определяет нумерацию $\mathscr{F}_{1}, \ldots, \mathscr{F}_{k}$ этих компонент.

Зафиксируем эту нумерацию. Тогда в последовательности точек пересечения кривой $\gamma$ с фронтом $\mathscr{F}$ можно выбрать подпоследовательность $x_{1}, x_{2}^{1}, x_{2}^{2}, \ldots$, $x_{k-1}^{1}, x_{k-1}^{2}, x_{k}$, такую, что

$$
x_{1} \in \mathscr{F}_{1}, \quad x_{k} \in \mathscr{F}_{k}, \quad x_{i}^{1} \in \mathscr{F}_{i}, \quad x_{i}^{2} \in \bigcup_{j=1}^{i} \mathscr{F}_{j}, \quad i=2, \ldots, k-1,
$$

а открытые дуги $\left(x_{1}, x_{2}^{1}\right),\left(x_{2}^{2}, x_{3}^{1}\right), \ldots,\left(x_{k-2}^{2}, x_{k-1}^{1}\right),\left(x_{k-1}^{2}, x_{k}\right)$ кривой $\gamma$ с концами в указанных точках не пересекают $\mathscr{F}$.

Отсюда следует, что существуют $C^{\infty}$-вложенные кривые $\gamma_{i}:[a, b] \rightarrow \mathbb{R}^{n}, i=$ $1, \ldots, k-1$, такие, что

(1) каждая кривая $\gamma_{i}$ пересекает фронт $\mathscr{F}$ ровно в двух гладких точках $P_{i}^{1}, P_{i}^{2}$, причем трансверсально;

(2) $P_{i}^{1}=\gamma_{i}\left(t_{1}\right) \in \bigcup_{j=1}^{i} \mathscr{F}_{j}$, a $P_{i}^{2}=\gamma_{i}\left(t_{2}\right) \in \mathscr{F}_{i+1}$, где $a<t_{1}<t_{2}<b$; 
(3) кривые $\gamma_{1}, \ldots, \gamma_{k-1}$ попарно не пересекаются.

Возьмем произвольное $\varepsilon>0$ и рассмотрим $\varepsilon$-окрестности $U_{i}$ кривых $\gamma_{i}$ в $\mathbb{R}^{n}$. Если $\varepsilon$ достаточно мало, то замыкания $\bar{U}_{1}, \ldots, \bar{U}_{k-1}$ этих окрестностей попарно не пересекаются; каждое пересечение $\bar{U}_{i} \cap \mathscr{F}$ состоит только из гладких точек фронта $\mathscr{F}$ и имеет ровно две связные компоненты $D_{i}^{1} \ni P_{i}^{1}, D_{i}^{2} \ni P_{i}^{2}$; подмножества $D_{i}^{1}, D_{i}^{2} \subset \mathbb{R}^{n}$ являются $C^{\infty}$-вложенными замкнутыми $(n-1)$-мерными дисками с гладкими краями $\partial D_{i}^{1}, \partial D_{i}^{2}$ (диффеоморфными $(n-2)$-мерной сфере $\left.S^{n-2}\right)$; каждая разность $\partial U_{i} \backslash\left(\partial D_{i}^{1} \cup \partial D_{i}^{2}\right)$, где $\partial U_{i}$ - граница окрестности $U_{i}$, имеет три связные компоненты; две из этих компонент гомеоморфны открытым $(n-1)$-мерным дискам, а замыкание $C_{i}$ третьей является $C^{\infty}$-вложенным цилиндром $S^{n-2} \times\left[t_{1}, t_{2}\right]$.

Следовательно, искомый фронт $\widetilde{\mathscr{F}}$ можно получить из множества

$$
\left[\mathscr{F} \backslash \bigcup_{i=1}^{k-1}\left(D_{i}^{1} \cup D_{i}^{2}\right)\right] \cup\left(\bigcup_{i=1}^{k-1} C_{i}\right)
$$

путем стандартного сглаживания (использующего разбиение единицы) в малых окрестностях сфер $\partial D_{i}^{1}, \partial D_{i}^{2}, i=1, \ldots, k-1$. Предложение 1 доказано.

ЗАмечАниЕ. Фронт $\widetilde{\mathscr{F}}$ является связной суммой фронтов $\mathscr{F}_{1}, \ldots, \mathscr{F}_{k}$ (см. [2]). Это суммирование проведено так, чтобы фронт $\widetilde{\mathscr{F}}$ обладал нужными нам свойствами.

ПреДЛОЖенИЕ 2. Для любого $\mathscr{A}=A_{\mu_{1}}+\cdots+A_{\mu_{m}}$, такого, что $\mu_{1}+\cdots+\mu_{m}$ $=n>1$, существует неприводимый компактный устойчивый фронт $\Sigma(\mathscr{A})$ коранга 1 в $\mathbb{R}^{n}$, который имеет ровно две особенности типа $\mathscr{A}$ и не имеет других изолированных особенностей.

ДоказАтельСтво. Рассмотрим подмножество $\Sigma$ в пространстве

$$
\mathbb{R}^{n+1}=\left\{\left(\lambda_{0}^{1}, \lambda_{1}^{1}, \ldots, \lambda_{\mu_{1}-1}^{1}, \ldots, \lambda_{0}^{m}, \lambda_{1}^{m}, \ldots, \lambda_{\mu_{m}-1}^{m}, \lambda_{*}\right)\right\},
$$

образованное точками, для которых хотя бы один из многочленов

$$
t^{\mu_{i}+1}+\lambda_{\mu_{i}-1}^{i} t^{\mu_{i}-1}+\cdots+\lambda_{1}^{i} t+\lambda_{0}^{i}, \quad i=1, \ldots, m,
$$

имеет кратный вещественный корень. Это подмножество является устойчивым фронтом коранга 1.

Зафиксируем в $\mathbb{R}^{n+1}$ стандартную сферу $S^{n}$ радиуса $R>0$ с центром в нуле. Пересечение фронта $\Sigma$ с этой сферой является компактным устойчивым фронтом коранга 1 на $S^{n}$. Этот фронт имеет ровно две изолированные особенности, и обе они являются особенностями типа $\mathscr{A}$.

Действительно, прямая $\lambda_{0}^{1}=\cdots=\lambda_{\mu_{m}-1}^{m}=0$ состоит из особенностей типа $\mathscr{A}$ фронта $\Sigma$ и является единственным одномерным стратом минимальной $C^{\infty}$-стратификации этого фронта. Вместе с любой своей точкой $\left(\lambda_{0}^{1}, \ldots, \lambda_{\mu_{m}-1}^{m}\right.$, $\left.\lambda_{*}\right) \neq 0$ каждый страт фронта $\Sigma$ содержит всю кривую $\gamma:(0,+\infty) \rightarrow \mathbb{R}^{n+1}$,

$$
\gamma: s \mapsto\left(\lambda_{0}^{1} s^{\mu_{1}+1}, \lambda_{1}^{1} s^{\mu_{1}}, \ldots, \lambda_{\mu_{1}-1}^{1} s^{2}, \ldots, \lambda_{0}^{m} s^{\mu_{m}+1}, \lambda_{1}^{m} s^{\mu_{m}}, \ldots, \lambda_{\mu_{m}-1}^{m} s^{2}, \lambda_{*} s\right) .
$$

Эта кривая трансверсально пересекает сферу $S^{n}$, причем ровно в одной точке. Следовательно, сфера $S^{n}$ трансверсальна каждому страту фронта $\Sigma$ и пересекает страт особенностей типа $\mathscr{A}$ ровно в двух точках. 
Остается воспользоваться стереографической проекцией гиперплоскости

$$
\lambda_{0}^{1}+\lambda_{0}^{2}+\cdots+\lambda_{0}^{m}=0
$$

на сферу $S^{n}$ без точки $P$, у которой $\lambda_{0}^{1}=\lambda_{0}^{2}=\cdots=\lambda_{0}^{m}=-R / \sqrt{m}$, а все остальные координаты равны нулю (точка $P$ служит полюсом проекции). Прообраз фронта $\Sigma \cap S^{n}$ относительно указанной проекции является фронтом в $\mathbb{R}^{n}$, который обладает всеми нужными свойствами, кроме неприводимости. Предложение 2 следует теперь из предложения 1.

ПреДЛОЖКЕНИЕ 3. Для любого $\mathscr{A}=A_{\mu_{1}}+\cdots+A_{\mu_{m}}$, такого, что $1<\mu_{1}+$ $\cdots+\mu_{m}=c \leqslant n$, существует неприводимый компактный устойчивый фронт $\Sigma_{n}(\mathscr{A})$ коранга 1 в $\mathbb{R}^{n}$, обладающий следующими свойствами:

(1) бронт $\Sigma_{n}(\mathscr{A})$ не имеет особенностей коразмерности выше с, а все его особенности коразмерности с являются особенностями типа $\mathscr{A}$;

(2) многообразие, образованное особенностями типа $\mathscr{A}$ бронта $\Sigma_{n}(\mathscr{A})$, диббеоморбно несвязному обгединению двух сфер размерности $n-c$.

ДокАЗАтЕЛЬСтво. Если $c<n$, то искомый фронт диффеоморфен фронту $\Sigma(\mathscr{A}) \times S^{n-c}$ в прямом произведении $\mathbb{R}^{c} \times S^{n-c}$, где $S^{n-c}-$ сфера размерности $n-c$, а $\Sigma(\mathscr{A})$ - неприводимый компактный устойчивый фронт коранга 1 в $\mathbb{R}^{c}$, который имеет ровно две особенности типа $\mathscr{A}$ и не имеет других изолированных особенностей (такой фронт существует согласно предложению 2). Если же $c=n$, то предложение 3 получается из предложения 2 с помощью предложения 1.

4. Доказательство теоремы 2. При $n=1$ это утверждение очевидно. Поэтому везде далее $n>1$. Кроме того, мы будем считать, что список $\mathscr{A}^{1}, \ldots, \mathscr{A}^{N}$ сформирован по неубыванию коразмерностей $\operatorname{codim} \mathscr{A}^{i}$. В частности, $\mathscr{A}^{1}=A_{1}$ при нечетном $n$ и $\mathscr{A}^{1}=2 A_{1}$, если $n$ четное.

Зафиксируем фронты $\Sigma_{n}\left(\mathscr{A}^{2}\right), \ldots, \Sigma_{n}\left(\mathscr{A}^{N}\right)$ в $\mathbb{R}^{n}$, обладающие свойствами (1)-(2) из предложения 3 для $\mathscr{A}=\mathscr{A}^{2}, \ldots, \mathscr{A}^{N}$ соответственно. Через $\Sigma_{n}\left(\mathscr{A}^{1}\right)$ обозначим аналогичный фронт для $\mathscr{A}=\mathscr{A}^{1}$, если $n$ четное, и $(n-1)$-мерный тор, $C^{\infty}$-вложенный в $\mathbb{R}^{n}$, если $n$ нечетное.

Пусть $\chi_{i, j}$ - эйлерова характеристика многообразия, образованного особенностями типа $\mathscr{A}^{j}$ фронта $\Sigma_{n}\left(\mathscr{A}^{i}\right)$, где $i, j=1, \ldots, N$. Зафиксируем произвольные целые неотрицательные числа $k_{1}, \ldots, k_{N}$, такие, что $k_{1}^{2}+\cdots+k_{N}^{2} \neq 0$. Для любого $i=1, \ldots, N$ построим $k_{i}$ фронтов $\mathscr{F}_{i}^{l}, l=1, \ldots, k_{i}$, в $\mathbb{R}^{n}$, диффеоморфных фронту $\Sigma_{n}\left(\mathscr{A}^{i}\right)$ и таких, что каждый фронт $\mathscr{F}_{i}^{l}$ расположен в одном из $k_{1}+\cdots+k_{N}$ попарно не пересекающихся открытых шаров в $\mathbb{R}^{n}$.

$\mathrm{K}$ фронтам $\mathscr{F}_{i}^{l}, i=1, \ldots, N, l=1, \ldots, k_{i}$, применим предложение 1 . Согласно этому утверждению, существует неприводимый компактный устойчивый фронт $\mathscr{F}$ коранга 1 в $\mathbb{R}^{n}$, такой, что

(1) эйлерова характеристика многообразия $\left(\mathscr{A}^{j}\right) \mathscr{F}$ особенностей типа $\mathscr{A}^{j}$ фронта $\mathscr{F}$ равна $\sum_{i=1}^{N} k_{i} \chi_{i, j}$ для любого $j=2, \ldots, N$;

(2) эйлерова характеристика многообразия $\left(\mathscr{A}^{1}\right) \mathscr{F}$ особенностей типа $\mathscr{A}^{1}$ фронта $\mathscr{F}$ равна $\sum_{i=1}^{N} k_{i}\left[\chi_{i, 1}-\chi\left(S^{n-1}\right)\right]+\chi\left(S^{n-1}\right)$, где $\chi\left(S^{n-1}\right)$ - эйлерова характеристика $(n-1)$-мерной сферы. 
Рассмотрим полугруппу в $\mathbb{R}^{N}$, состоящую из векторов

$$
\left(\sum_{i=1}^{N} k_{i}\left[\chi_{i, 1}-\chi\left(S^{n-1}\right)\right], \sum_{i=1}^{N} k_{i} \chi_{i, 2}, \ldots, \sum_{i=1}^{N} k_{i} \chi_{i, N}\right),
$$

соответствующих всевозможным упорядоченным наборам целых неотрицательных чисел $\left(k_{1}, \ldots, k_{N}\right)$. Эта полугруппа порождена векторами

$$
v_{i}=\left(\chi_{i, 1}-\chi\left(S^{n-1}\right), \chi_{i, 2}, \ldots, \chi_{i, N}\right), \quad i=1, \ldots, N .
$$

ПрЕДЛОЖЕНИЕ 4. Векторы $v_{1}, \ldots, v_{N}$ линейно независимы.

Доказательство. Рассмотрим $(N \times N)$-матрицу $M$, строками которой служат векторы $v_{1}, \ldots, v_{N}$. Матрица $M$ является нижнетреугольной $\left(\chi_{i, j}=0\right.$ при всех $j>i)$. На ее главной диагонали стоят ненулевые числа: $\chi_{1,1}-\chi\left(S^{n-1}\right)=$ -2 , если $\mathscr{A}^{1}=A_{1}$, и $\chi_{i, i}=2 \chi\left(S^{n-\operatorname{codim} \mathscr{A}^{i}}\right)=4$ для любого $\mathscr{A}^{i} \neq A_{1}$ (напомним, что $\left.\operatorname{codim} \mathscr{A}^{i} \equiv n \bmod 2\right)$. Таким образом, $\operatorname{det} M \neq 0$. Предложение 4 доказано.

Теорема 2 следует теперь из предложения 4.

Автор благодарен В. И. Арнольду и М. Э. Казаряну за полезные обсуждения и замечания.

\section{ЛитератУРА}

1. Арнольд В. И., Варченко А. Н., Гусейн-Заде С. М. Особенности дифференцируемых отображений, т. 1. Наука, М., 1982.

2. Arnold V. I. Plane curves, their invariants, perestroikas and classifications. Adv. Sov. Math., 21, 33-91 (1994).

3. Васильев B. А. Лагранжевы и лежандровы характеристические классы. МЦНМО, M., 2000.

4. Седъх В. Д. Разрешение особенностей коранга 1 фронта общего положения. Функц. анализ и его прил., 37, вып. 2, 52-64 (2003).

Российский государственный университет нефти и газа им. И. М. Губкина

Поступило в редакцию 4 апреля 2003 г. 